\title{
ON THE IRREDUCIBILITY OF PSEUDOVARIETIES OF SEMIGROUPS
}

\author{
J. ALMEIDA AND O. KLÍMA
}

\begin{abstract}
We show that, for every pseudovariety of groups $H$, the pseudovariety $\overline{\mathrm{H}}$, consisting of all finite semigroups all of whose subgroups lie in $\mathrm{H}$, is irreducible for join and the Mal'cev and semidirect products.
\end{abstract}

\section{INTRODUCTION}

Since the establishment of Eilenberg's correspondence between varieties of regular languages and pseudovarieties of semigroups [5], the theory of finite semigroups has evolved mostly in the direction of their classification in pseudovarieties. The most recent account on this topic is [15], which contains a wealth of results, centered on the Krohn-Rhodes complexity theory, but not limited to it. The typical problem, motivated by the origins of this research area, consists in determining whether the membership problem for a given pseudovariety is decidable. The difficulty lies in the fact that, very often, pseudovarieties are given by generators, rather than by characteristic structural properties of their members. The generators are often obtained by applying some natural algebraic construction to members of given pseudovarieties. For instance, the direct and semidirect products of semigroups lead respectively to the join and semidirect product of pseudovarieties, while the existence of a congruence whose idempotent classes lie in a given pseudovariety and whose quotient lies in another pseudovariety leads to the Mal'cev product. The interest in such operators on pseudovarieties is that they allow to decompose, in the pseudovariety sense, complicated finite semigroups in terms of simpler ones. For example, the Krohn-Rhodes decomposition theory concerns building arbitrary finite semigroups from finite simple groups and finite aperiodic semigroups using semidirect products.

Thus, a key ingredient in the theory of pseudovarieties of semigroups is to break them up, when possible, into simpler pseudovarieties using natural operators. There are two ways in which this might be achieved: through a finite decomposition, or through an iterated decomposition, providing a filtration of the pseudovariety in terms of subpseudovarieties which admit finite decompositions, as in the Krohn-Rhodes complexity theory.

In this paper, we improve on earlier results of Margolis, Sapir and Weil [8] and Rhodes and Steinberg [14, 15] concerning the pseudovarieties of the form $\overline{\mathrm{H}}$, consisting of all finite semigroups all of whose subgroups lie in a

\footnotetext{
2010 Mathematics Subject Classification. Primary 20M07; Secondary 20M05, 20M35.

Key words and phrases. pseudovariety, profinite semigroup, encoding, Malcev product, semidirect product, join, irreducibility, pseudoword.

Corresponding author: J. Almeida.
} 
given pseudovariety of groups $\mathrm{H}$. In [8], Koryakov's embedding approach [6] is improved to show that $\overline{\mathrm{H}}$ is finitely indecomposable in terms of Mal'cev and semidirect products and joins, provided $\mathrm{H}$ is closed under semidirect product. In [14], a stronger form of join indecomposability (called finite join irreducibility) is established for $\overline{\mathrm{H}}$ in case $\mathrm{H}$ contains some non-nilpotent group, the technique being the construction of so-called Kovács-Newman semigroups. In both works, it is proposed as an open problem to determine whether every pseudovariety of the form $\overline{\mathrm{H}}$ is join indecomposable. This also appears in [15] as Problem 47.

We give an affirmative solution to those problems. Our approach is similar to that of [8] but uses a Rees matrix-like construction to obtain an improved embedding of free pro- $\overline{\mathrm{H}}$ semigroups, which does not require that $\mathrm{H}$ be closed under semidirect product. This allows us to use the arguments of 8 ] to show that $\overline{\mathrm{H}}$ is indecomposable, in the stronger sense, with respect to both join and the Mal'cev and semidirect products. In fact, we show that every pseudovariety that is closed under our construction is join indecomposable in the weaker sense. The construction leads to a new operator at the level of pseudovarieties, which we call the bullet. We have not found any pseudovarieties other than those of the form $\overline{\mathrm{H}}$ which are closed under bullet.

\section{Preliminaries}

We assume familiarity with the basic theory of pseudovarieties of semigroups, including the role played by free profinite semigroups, in particular through Reiterman's theorem [10, defining pseudovarieties by pseudoidentities. The reader is referred to [1, 2, 15] for a few alternative introductions to this subject.

For a pseudovariety $\mathrm{V}, \bar{\Omega}_{A} \mathrm{~V}$ and $\bar{\Omega}_{n} \mathrm{~V}$ denote the pro- $\mathrm{V}$ semigroups freely generated respectively by the set $A$ and a set of cardinality $n$. Elements of such semigroups will be called pseudowords.

We adopt the usual conventions for semigroup pseudoidentities such as that $u=1$ and $u=0$ are, respectively, abbreviations of the pseudoidentities $u x=x=x u$ and $u x=u=x u$, where $x$ is a variable that does not occur in $u$.

For the reader's convenience, the following is a catalog of pseudovarieties of finite semigroups that play a role in this paper. For each of them, besides a, sometimes incomplete, verbal description, a well-known definition in terms of pseudoidentities is also provided.

- I: trivial $(\llbracket x=y \rrbracket)$.

- LZ: left zero $(\llbracket x y=x \rrbracket)$.

- RZ: right zero $(\llbracket x y=y \rrbracket)$.

- RB: rectangular bands $(\llbracket x y x=x \rrbracket)$.

- A: aperiodic $\left(\llbracket x^{\omega+1}=x^{\omega} \rrbracket=\mathrm{B}_{\omega, 1}\right)$.

- S: all $\left(\llbracket x=x \rrbracket=\mathrm{B}_{\omega, \omega}\right)$.

- $\mathrm{N}_{2}$ : null (nilpotency index at most $2, \llbracket x y=0 \rrbracket$ ).

- D: definite $\left(\llbracket x y^{\omega}=y^{\omega} \rrbracket\right)$.

- $\mathrm{K}_{n}$ : reverse definite of index $n\left(\llbracket x_{1} \cdots x_{n} y=x_{1} \cdots x_{n} \rrbracket\right)$.

- $\mathrm{K}$ : reverse definite $\left(\llbracket x^{\omega} y=x^{\omega} \rrbracket\right)$. 
- Ab: Abelian groups $\left(\llbracket x^{\omega}=1, x y=y x \rrbracket\right)$.

Let $\mathrm{V}$ be a pseudovariety of semigroups. A homomorphism $\varphi: S \rightarrow T$ between two finite semigroups $S$ and $T$ is said to be a V-homomorphism if $\varphi^{-1}(e) \in \mathrm{V}$ for every idempotent $e \in T$.

We also need the following operators on pseudovarieties of semigroups:

- Mal'cev product: $\mathrm{V}(\mathrm{m} \mathrm{W}$ is the pseudovariety generated by all finite semigroups $S$ for which there is a $\mathrm{V}$-homomorphism $\varphi: S \rightarrow T$ into a semigroup $T \in \mathrm{W}$;

- semidirect product: $\mathrm{V} * \mathrm{~W}$ is the pseudovariety generated by all semidirect products of the form $S * T$ with $S \in \mathrm{V}$ and $T \in \mathrm{W}$;

- bar: for a pseudovariety $\mathrm{H}$ (of groups), $\overline{\mathrm{H}}$ is the pseudovariety consisting of all finite semigroups all of whose subgroups belong to $\mathrm{H}$;

- localization: for a pseudovariety V, LV consists of all finite semigroups $S$ such that, for every idempotent $e$ in $S$, the local subsemigroup $e S e$ belongs to $\mathrm{V}$.

It is well known that $\mathrm{V} * \mathrm{~W}$ is also generated by the wreath products of the form $S \circ T$ with $S \in \mathrm{V}$ and $T \in \mathrm{W}$, a fact which may be used to deduce that the semidirect product of pseudovarieties is associative.

The Basis Theorem for the Mal'cev product of pseudovarieties [9] states that, if $\mathrm{V}=\llbracket u_{i}\left(x_{1}, \ldots, x_{n_{i}}\right)=v_{i}\left(x_{1}, \ldots, x_{n_{i}}\right): i \in I \rrbracket$ then $\mathrm{V}(m \mathrm{~W}$ is defined by the pseudoidentities of the form $u_{i}\left(w_{1}, \ldots, w_{n_{i}}\right)=v_{i}\left(w_{1}, \ldots, w_{n_{i}}\right)$ where the $w_{j}$ are pseudowords such that $\mathrm{W}$ satisfies the pseudoidentities $w_{1}^{2}=w_{1}=\cdots=w_{n_{i}}(i \in I)$.

\section{A ReEs matrix eXtension CONSTRUCtion}

Let $S$ and $T$ be semigroups and $f: S^{1} \rightarrow T^{1}$ be a function. The set

$$
M(S, T, f)=S \uplus S^{1} \times T^{1} \times S^{1}
$$

is endowed with the multiplication defined by the following formulas for all $s \in S, s_{i}, s_{i}^{\prime} \in S^{1}$, and $t, t^{\prime} \in T^{1}$ :

$$
\begin{aligned}
s_{1} \cdot s_{2} & =s_{1} s_{2} \\
s \cdot\left(s_{1}, t, s_{2}\right) & =\left(s s_{1}, t, s_{2}\right) \\
\left(s_{1}, t, s_{2}\right) \cdot s & =\left(s_{1}, t, s_{2} s\right) \\
\left(s_{1}, t, s_{2}\right) \cdot\left(s_{1}^{\prime}, t^{\prime}, s_{2}^{\prime}\right) & =\left(s_{1}, t f\left(s_{2} s_{1}^{\prime}\right) t^{\prime}, s_{2}^{\prime}\right) .
\end{aligned}
$$

The $S^{1} \times T^{1} \times S^{1}$ portion of $M(S, T, f)$, which constitutes an ideal, is a special case of the well-known Rees matrix semigroup construction that can be found in most of the literature on the algebraic theory of semigroups (see, for instance, [3]). The semigroup $M(S, T, f)$ has recently been used in [4] for rather different purposes. Similar constructions have been extensively used in the synthesis theory of Rhodes [13, 11, 12].

The following lemma contains some preliminary observations about this algebraic structure.

Lemma 3.1. The set $M(S, T, f)$ is a semigroup for the above operation. All its subgroups are isomorphic to subgroups of either $S$ or $T$. 
Proof. From the definition, it is clear that $S$ is a subsemigroup and that, its complement, the subset $R=S^{1} \times T^{1} \times S^{1}$ constitutes a Rees matrix subsemigroup. Moreover, the formulas indicate that $S$ acts both on the left and on the right of $R$, respectively by left multiplication on the first component and right multiplication on the third component. Therefore, the two actions commute. Hence, the only case of the associativity law that remains to be considered is

$$
\left(\left(s_{1}, t, s_{2}\right) \cdot s\right) \cdot\left(s_{1}^{\prime}, t^{\prime}, s_{2}^{\prime}\right)=\left(s_{1}, t, s_{2}\right) \cdot\left(s \cdot\left(s_{1}^{\prime}, t^{\prime}, s_{2}^{\prime}\right)\right),
$$

and it is easily checked that both sides are equal to $\left(s_{1}, t f\left(s_{2} s s_{1}^{\prime}\right) t^{\prime}, s_{2}^{\prime}\right)$.

Let $H$ be a subgroup of $M(S, T, f)$. Since $R$ is an ideal, $H$ must be contained in either $S$ or $R$, and it suffices to consider the latter case. Let $\left(s_{1}, t, s_{2}\right)$ be the idempotent of $H$. Then $t f\left(s_{2} s_{1}\right) t=t$ and $e=t f\left(s_{2} s_{1}\right)$ is an idempotent of $T^{1}$. Let $G$ be the maximal subgroup of $T^{1}$ containing $e$. Consider the mapping $\varphi: H \rightarrow T^{1}$ which sends each element $\left(s_{1}, t^{\prime}, s_{2}\right)$ to $t^{\prime} f\left(s_{2} s_{1}\right)$. It is routine to check that $\varphi$ is an injective homomorphism which takes its values in $G$.

Given two pseudovarieties of semigroups $\mathrm{U}$ and $\mathrm{V}$, we denote by $\mathrm{U} \bullet \mathrm{V}$ the pseudovariety generated by all semigroups of the form $M(S, T, f)$, with $S \in \mathrm{U}$ and $T \in \mathrm{V}$. For lack of a better name, we call bullet this operation on pseudovarieties. We say that $\mathrm{V}$ is a bullet idempotent if $\mathrm{V} \bullet \mathrm{V}=\mathrm{V}$. Note that, in view of Lemma 3.1, every pseudovariety of the form $\overline{\mathrm{H}}$ is a bullet idempotent, provided $\mathrm{H}$ is a pseudovariety of groups.

The following embedding theorem is the core of our irreducibility results. It may be viewed as a strong generalization of the coding statement given by [8, Proposition 2.4].

Theorem 3.2. Let $\bigvee$ be a pseudovariety which is a bullet idempotent. Let $A$ and $B$ be finite sets and suppose that $\theta: B \rightarrow \bar{\Omega}_{A} \mathrm{~V}$ is an injective function. Then the unique continuous homomorphism $\psi: \bar{\Omega}_{B} \mathrm{~V} \rightarrow \bar{\Omega}_{A \uplus\{z\}} \bigvee$ such that $\psi(b)=\theta(b) z$ is injective.

Proof. Arguing by contradiction, let $u, v \in \bar{\Omega}_{B} \bigvee$ be such that $\psi(u)=\psi(v)$ and $u \neq v$. Let $\tau: \bar{\Omega}_{B} \bigvee \rightarrow T$ be a continuous homomorphism into a semigroup $T$ from $\mathrm{V}$ such that $\tau(u) \neq \tau(v)$. Let $\sigma: \bar{\Omega}_{A} \mathrm{~V} \rightarrow S$ be a continuous homomorphism into a semigroup $S$ from $\mathrm{V}$ such that the mapping $\sigma \circ \theta$ is injective. Let $f: S^{1} \rightarrow T^{1}$ be the function defined by $f(\sigma(\theta(b)))=\tau(b)$, and $f(s)=1$ for all other $s \in S^{1}$. Since $\mathrm{V}$ is a bullet idempotent, there is a unique continuous homomorphism $\varphi: \bar{\Omega}_{A \uplus\{z\}} \bigvee \rightarrow M(S, T, f)$ such that $\varphi(a)=\sigma(a)$ for $a \in A$ and $\varphi(z)=(1,1,1)$. We claim that the equality

$$
\varphi(z \psi(w))=(1, \tau(w), 1)
$$

holds for every $w \in \bar{\Omega}_{B} \mathrm{~V}$. This will complete the proof since it contradicts the initial assumptions $\psi(u)=\psi(v)$ and $\tau(u) \neq \tau(v)$.

Since the mappings $\varphi, \psi$ and $\tau$ are continuous, it suffices to prove (1) in case $w \in B^{+}$, which we establish by induction on $|w|$. Suppose first that $w=b \in B$. Then we have

$$
\begin{aligned}
\varphi(z \psi(b)) & =\varphi(z \theta(b) z)=\varphi(z) \varphi(\theta(b)) \varphi(z) \\
& =(1,1,1) \sigma(\theta(b))(1,1,1)=(1, f(\sigma(\theta(b))), 1)=(1, \tau(b), 1) .
\end{aligned}
$$


Suppose next that $b \in B$ and $w \in B^{+}$satisfies (11). Then we may compute

$$
\begin{aligned}
\varphi(z \psi(b w)) & =\varphi(z \theta(b) z \psi(w))=\varphi(z) \varphi(\theta(b)) \varphi(z \psi(w)) \\
& =(1,1,1) \sigma(\theta(b))(1, \tau(w), 1)=(1, f(\sigma(\theta(b))) \tau(w), 1) \\
& =(1, \tau(b) \tau(w), 1)=(1, \tau(b w), 1),
\end{aligned}
$$

which completes the induction step and establishes the claim.

Recall that a pseudovariety $\mathrm{V}$ is monoidal if it contains the monoid $S^{1}$ whenever it contains the semigroup $S$.

Lemma 3.3. Every bullet idempotent is monoidal.

Proof. Let $T$ be an arbitrary semigroup from $\mathrm{V}$ and consider the associated semigroup $U=M(\{1\}, T, f)$, where $f:\{1\} \rightarrow T^{1}$ maps 1 to 1 . Then $\{1\} \times T^{1} \times\{1\}$ is a subsemigroup of $U$ which is isomorphic with $T^{1}$. Hence, $T^{1}$ belongs to $\mathrm{V} \bullet \mathrm{V}$ and, therefore, also to $\mathrm{V}$.

We say that a pseudovariety $\mathrm{V}$ has finite index if it satisfies a pseudoidentity of the form $x^{m}=x^{m+\omega}$ with $m$ a positive integer. In this case, the smallest such $m$ is called the index of $\mathbf{V}$. If there is no such $m$, then $\mathrm{V}$ is said to have infinite index.

Lemma 3.4. Every bullet idempotent contains $\mathrm{LI}$ and, in particular, it has infinite index.

Proof. Let V be a bullet idempotent. As a pseudovariety, it must contain the trivial semigroup $I=\{1\}$ and, therefore, also the two-element semilattice $S=M(I, I, f)$. For the constant mapping $f$ with value 0 , the subsemigroup of $M(S, S, f)$ given by $S \times\{0\} \times S$ is a $2 \times 2$ rectangular band, while the subsemigroup $\{1\} \times S \times\{1\}$ is a two-element null semigroup. Hence $\mathrm{V}$ contains $\mathrm{RB}=\mathrm{LZ} \vee \mathrm{RZ}$ and $\mathrm{N}_{2}$.

Let $A$ be a finite alphabet and let $K_{n}=\bar{\Omega}_{A} \mathrm{~K}_{n}$. It is well known that $K_{n}$ may be represented by the set $A^{\leq n}$ of nonempty words of length at most $n$, with multiplication given by concatenation followed by truncation to the prefix of length $n$ if the resulting word has length greater than $n$. By induction on $n$, we prove that $K_{n} \in \mathrm{V}$. The case of $n=1$ follows from the above since $\mathrm{K}_{1}=\mathrm{LZ}$. Assuming that $K_{n} \in \mathrm{V}$, consider the semigroup $U=M\left(\bar{\Omega}_{A} \mathrm{~N}_{2}, K_{n}, f\right)$, where $f:\left(\bar{\Omega}_{A} \mathrm{~N}_{2}\right)^{1} \rightarrow \mathrm{K}_{n}$ maps each free generator $a \in A$ of $\bar{\Omega}_{A} \mathrm{~N}_{2}$ to the corresponding free generator of $K_{n}$. Noting that $\bar{\Omega}_{A} \mathrm{~N}_{2}=A \uplus\{0\}$, the set $T=A \times K_{n} \times\{1\}$ is a subsemigroup of $U$ and the mapping $T \rightarrow K_{n+1}$ which sends each triple $(a, w, 1) \in T$ to $a w$ is an onto homomorphism, we deduce that $K_{n+1} \in \mathrm{V}$.

The preceding paragraph entails that $\mathrm{K}$ is contained in $\mathrm{V}$. Dually, so is $\mathrm{D}$ and, therefore so is $\mathrm{LI}=\mathrm{K} \vee \mathrm{D}$.

We adopt the same terminology as in [15, Definition 6.1.5] for various irreducibility notions in a lattice. In particular, we say that

- a pseudovariety $\mathrm{V}$ is strictly finite join irreducible (sfji) if $\mathrm{V}=\mathrm{U} \vee \mathrm{W}$ implies $\mathrm{V}=\mathrm{U}$ or $\mathrm{V}=\mathrm{W}$;

- a pseudovariety $\mathrm{V}$ is finite join irreducible (fji) if $\mathrm{V} \subseteq \mathrm{U} \vee \mathrm{W}$ implies $\mathrm{V} \subseteq \mathrm{U}$ or $\mathrm{V} \subseteq \mathrm{W}$. 
Theorem 3.5. Every bullet idempotent is sfji.

Proof. Let $\mathrm{V}$ be a bullet idempotent and let $\mathrm{U}$ and $\mathrm{W}$ be pseudovarieties of semigroups such that $\mathrm{U} \vee \mathrm{W}=\mathrm{V}$. We claim that $\mathrm{V}$ must be contained (and therefore be equal) to at least one of $U$ and W. Otherwise, by Reiterman's theorem, there are pseudoidentities $u_{1}=u_{2}$ and $v_{1}=v_{2}$ such that $\mathrm{U}$ satisfies $u_{1}=u_{2}, \mathrm{~W}$ satisfies $v_{1}=v_{2}$, and $\mathrm{V}$ fails both pseudoidentities. We may assume that each of the pseudoidentities $u_{1}=u_{2}$ and $v_{1}=v_{2}$ involves the minimum possible number of variables so that, in particular, every pseudoidentity which is obtained from them by identifying variables is valid in $\mathrm{V}$. Without loss of generality, we further assume that the number of variables $n$ involved in $u_{1}=u_{2}$ is at least the number of variables involved in $v_{1}=v_{2}$.

Consider first the case where $n \geq 2$ and let $B=\left\{x_{1}, \ldots, x_{n}\right\}$ be the set of variables involved in the pseudoidentity $u_{1}=u_{2}$. We may assume that the pseudoidentity $v_{1}=v_{2}$ is written on a disjoint set $C$ of variables and we let $A=B \cup C$. We further consider a new variable $z$. Since $\vee$ contains all finite rectangular bands by Lemma 3.4, we deduce that the mapping $\theta: B \rightarrow \bar{\Omega}_{A} \mathrm{~V}$ sending $x_{i}$ to $v_{i}$ for $i=1,2$ and fixing all other $x_{j}$ is injective. By Theorem 3.2, the unique continuous homomorphism $\psi: \bar{\Omega}_{B} \mathrm{~V} \rightarrow \bar{\Omega}_{A \cup\{z\}} \mathrm{V}$ such that $\psi\left(x_{i}\right)=\theta\left(x_{i}\right) z$ is also injective. Since the pseudoidentity $u_{1}=u_{2}$ fails in $\mathrm{V}$, it follows that so does the pseudoidentity

$$
u_{1}\left(v_{1} z, v_{2} z, x_{3} z, \ldots, x_{n} z\right)=u_{2}\left(v_{1} z, v_{2} z, x_{3} z, \ldots, x_{n} z\right) .
$$

Note the pseudoidentity (2) is valid in $\mathrm{U}$, being an obvious consequence of $u_{1}=u_{2}$. It is also valid in $\mathrm{W}$ since this pseudovariety satisfies $v_{1}=v_{2}$, so that W satisfies (2) if and only if it satisfies the pseudoidentity

$$
u_{1}\left(v_{1} z, v_{1} z, x_{3} z, \ldots, x_{n} z\right)=u_{2}\left(v_{1} z, v_{1} z, x_{3} z, \ldots, x_{n} z\right),
$$

which, by the assumption on the minimality of the number $n$, is valid in $\mathrm{V}$, whence also in $W$. Hence $U \vee W$ satisfies the pseudoidentity (2), while $V$ does not, which contradicts the assumption that $\mathrm{V}=\mathrm{U} \vee \mathrm{W}$.

It remains to consider the case where $n=1$. In this case, the pseudoidentity $u_{1}=u_{2}$ involves only one variable. Then, $u_{1}(x) u_{2}(y)=u_{2}(x) u_{1}(y)$ is still a pseudoidentity valid in $\mathrm{U}$. It fails in $\mathrm{V}$ since $\mathrm{V}$ is monoidal by Lemma 3.3. We may then apply basically the same argument as in the case $n \geq 2$ to the pseudoidentity $u_{1}(x) u_{2}(y)=u_{2}(x) u_{1}(y)$ playing the role of $u_{1}=u_{2}$. The only difference in the argument concerns the verification that the pseudoidentity (2) holds in W, which is now trivial since, in the presence of $v_{1}=v_{2}$, (2) is equivalent to $u_{1}\left(v_{1} z\right) u_{2}\left(v_{1} z\right)=u_{2}\left(v_{1} z\right) u_{1}\left(v_{1} z\right)$.

In view of Lemma 3.1. Theorem 3.5 applies to the pseudovarieties of the form $\overline{\mathrm{H}}$, which gives an affirmative solution to the first part of [15, Problem 47]. However, we prove in Section 4 that in fact $\bar{H}$ is fji, which provides an affirmative answer also to the second part of [15, Problem 47].

\section{AN IMPROVED FINITE JOIN IRREDUCIBILITY RESUlT}

In this section we prove that every pseudovariety of the form $\overline{\mathrm{H}}$ is irreducible not only for the join (fji) but also enjoys the analogous properties for both the Mal'cev and semidirect products. This improves the main results of 8 . 
Theorem 4.1. Let $\mathrm{H}$ be a pseudovariety of groups. If $\overline{\mathrm{H}}$ is contained in $\mathrm{V}(\mathrm{m} \mathrm{W}$, then it is contained in at least one of $\mathrm{V}$ and $\mathrm{W}$.

Proof. Suppose that $\overline{\mathrm{H}} \subseteq \mathrm{V} \otimes \mathrm{W}$ and that $\overline{\mathrm{H}}$ is contained in neither $\mathrm{V}$ nor $\mathrm{W}$. By Reiterman's theorem, there are pseudoidentities $u=v$ and $w=t$ that fail in $\overline{\mathrm{H}}$ but hold respectively in $\mathrm{V}$ and $\mathrm{W}$. By [ 6 , Theorem 1], we may assume that $u, v \in \bar{\Omega}_{2} \mathrm{~S}$. Let $A$ be a finite set such that $w, t \in \bar{\Omega}_{A} \mathrm{~S}$ and choose $z \notin A$. Note that the pseudoidentity

(3)

$$
u\left((w z)^{\omega}(t z)^{\omega},(w z)^{\omega}(t z w z)^{\omega}(t z)^{\omega}\right)=v\left((w z)^{\omega}(t z)^{\omega},(w z)^{\omega}(t z w z)^{\omega}(t z)^{\omega}\right)
$$

holds in $\mathrm{V}: \mathrm{W}$. Indeed, this pseudovariety is generated by all finite semigroups $S$ for which there exists a homomorphism $f: S \rightarrow T$ into a semigroup from $\mathrm{W}$ such that $f^{-1}(e) \in \mathrm{V}$ for every idempotent $e$ from $T$. For a continuous homomorphism $\varphi: \bar{\Omega}_{A} \mathrm{~S} \rightarrow S$, since $\mathrm{W}$ satisfies the pseudoidentity $w=t$ and $T \in \mathrm{W}, f\left(\varphi\left((w z)^{\omega}(t z)^{\omega}\right)\right)$ and $f\left(\varphi\left((w z)^{\omega}(t z w z)^{\omega}(t z)^{\omega}\right)\right)$ are the same idempotent $e$. Thus, $\varphi\left((w z)^{\omega}(t z)^{\omega}\right)$ and $\varphi\left((w z)^{\omega}(t z w z)^{\omega}(t z)^{\omega}\right)$ are both elements of the subsemigroup $f^{-1}(e)$ of $S$, which in turn belongs to $\mathrm{V}$. Hence $\varphi$ maps both sides of the pseudoidentity (3) to the same element of $S \mathbb{1}$

It remains to show that the pseudoidentity (3) fails in $\overline{\mathrm{H}}$. Indeed, it is shown in the proof of [8, Proposition 3.1] that, since the pseudovariety $\bar{H}$ is monoidal and it fails the pseudoidentity $u=v$, it also fails the pseudoidentity

$$
u\left(x^{\omega} y^{\omega}, x^{\omega}(y x)^{\omega} y^{\omega}\right)=v\left(x^{\omega} y^{\omega}, x^{\omega}(y x)^{\omega} y^{\omega}\right) .
$$

Let $p: \bar{\Omega}_{\{x, y\}} \mathrm{S} \rightarrow \bar{\Omega}_{\{x, y\}} \overline{\mathrm{H}}$ and $q: \bar{\Omega}_{A \cup\{z\}} \mathrm{S} \rightarrow \bar{\Omega}_{A \cup\{z\}} \overline{\mathrm{H}}$ be the natural continuous homomorphisms. To apply Theorem 3.2 , let $\theta:\{x, y\} \rightarrow \bar{\Omega}_{A} \overline{\mathrm{H}}$ be the function that maps $x$ to $q(w)$ and $y$ to $q(t)$. Note that $\theta$ is injective because the pseudoidentity $w=t$ fails in $\overline{\mathrm{H}}$. Let $\psi: \bar{\Omega}_{\{x, y\}} \overline{\mathrm{H}} \rightarrow \bar{\Omega}_{A \cup\{z\}} \overline{\mathrm{H}}$ be the resulting injective continuous homomorphism defined in Theorem 3.2 . Since, for $s \in \bar{\Omega}_{\{x, y\}} \mathrm{S}$,

$$
\psi\left(p\left(s\left(x^{\omega} y^{\omega}, x^{\omega}(y x)^{\omega} y^{\omega}\right)\right)\right)=q\left(s\left((w z)^{\omega}(t z)^{\omega},(w z)^{\omega}(t z w z)^{\omega}(t z)^{\omega}\right)\right)
$$

and $\psi$ is injective, from the fact that the pseudoidentity (4) fails in $\overline{\mathrm{H}}$ it follows that so does (3).

From Theorem 4.1, one may adapt the arguments used in [8, proofs of Corollaries 3.3 and 3.4] to deduce the following results. The adaptation consists in dropping the hypothesis that the pseudovariety of groups $\mathrm{H}$ is closed under extensions, and noting that in all cases the arguments yield finite irreducibility rather than just strict finite irreducibility. The short proofs are included for the sake of completeness.

Corollary 4.2. Let $\mathrm{H}$ be an arbitrary pseudovariety of groups. If $\overline{\mathrm{H}}$ is contained in a semidirect product $\mathrm{V} * \mathrm{~W}$, then it is contained in at least one of the factors $\mathrm{V}$ and $\mathrm{W}$.

Proof. By [15, Corollary 4.1.32], we have V*W $\subseteq$ LV $m$ W. From the hypothesis and Theorem 4.1, we deduce that $\overline{\mathrm{H}}$ is contained in at least one

\footnotetext{
${ }^{1}$ This argument, which is included here for the sake of completeness, is basically the easy part of the proof of the Pin and Weil Basis Theorem for Mal'cev products [9].
} 
of the Mal'cev factors LV and $W$. In the former case, since $\bar{H}$ is monoidal, it follows that it is contained in $\mathrm{V}$.

Corollary 4.3. If $\mathrm{H}$ is an arbitrary pseudovariety of groups, then $\overline{\mathrm{H}}$ is fji.

Proof. It suffices to note that $\mathrm{V} \vee \mathrm{W} \subseteq \mathrm{V} * \mathrm{~W}$ and apply Corollary 4.2 ,

The special case of Corollary 4.3 where $\mathrm{H}$ is a pseudovariety of groups containing some non-nilpotent group is part of [15, Corollary 7.4.23], which is based on the construction of so-called Kovács-Newman semigroups. Corollary 4.3 solves [15, Problem 47]. The strict version of that problem, as well the Mal'cev and semidirect products counterparts had already been proposed in [8]. The following result is an immediate application of strict finite join irreducibility of $\overline{\mathrm{H}}$.

Corollary 4.4. Let $\mathrm{H}$ be an arbitrary pseudovariety of groups. Then $\overline{\mathrm{H}}$ contains no maximal proper subpseudovariety.

Proof. Since no pseudoidentity of the form $x^{n}=x^{\omega+n}$ with $n$ a positive integer holds in $\overline{\mathrm{H}}$, this pseudovariety cannot be finitely generated. The result now follows from [15, Corollary 7.2.17], which states that every sfji pseudovariety that is not finitely generated has no proper maximal subpseudovariety.

For pseudovarieties of groups $\mathrm{H}$ containing $\mathrm{Ab}$, Corollary 4.4 had previously been proved by Margolis [7].

\section{Conclusion}

We have extended the irreducibility results on pseudovarieties of the form $\overline{\mathrm{H}}$ to the case of an arbitrary pseudovariety of groups $\mathrm{H}$, thereby proving the superfluousness of extra hypotheses that were previously considered in the literature. Theorem 3.5 actually establishes a weaker form of irreducibility, namely sfji, for possibly more general types of pseudovarieties, the bullet idempotents, but it remains open whether the theorem applies to any other pseudovarieties than those of the form $\overline{\mathrm{H}}$. More generally, it remains an open problem to determine all sfji and all fji pseudovarieties of semigroups.

Acknowledgments. Both authors were partially supported by the Ministry of Education of the Czech Republic under the project MSM 0021622409 and by the Grant 201/09/1313 of the Grant Agency of the Czech Republic.

The first author also acknowledges partial funding by the European Regional Development Fund, through the programme COMPETE, and by the Portuguese Government through FCT - Fundação para a Ciência e a Tecnologia, under the project PEst-C/MAT/UI0144/2011. This work has been carried out during the first author's sabbatical visit to the Department of Mathematics and Statistics of Masaryk University, whose hospitality and support is hereby gratefully acknowledged.

\section{REFERENCES}

1. J. Almeida, Finite semigroups and universal algebra, World Scientific, Singapore, 1995, English translation. 
2. __ Profinite semigroups and applications, Structural Theory of Automata, Semigroups and Universal Algebra (New York) (V. B. Kudryavtsev and I. G. Rosenberg, eds.), Springer, 2005, pp. 1-45.

3. A. H. Clifford and G. B. Preston, The algebraic theory of semigroups, vol. I, Amer. Math. Soc., Providence, R.I., 1961.

4. V. Diekert, M. Kufleitner, and P. Weil, Star-free languages are Church-Rosser congruential, Theor. Comp. Sci. (2012), 129-135.

5. S. Eilenberg, Automata, languages and machines, vol. B, Academic Press, New York, 1976.

6. I. O. Koryakov, Embeddings of pseudofree semigroups, Russian Math. (Iz. VUZ) 39 (1995), 53-59.

7. S. Margolis, On maximal pseudo-varieties of finite monoids and semigroups, Russian Math. (Iz. VUZ) 39 (1995), 60-64.

8. S. Margolis, M. Sapir, and P. Weil, Irreducibility of certain pseudovarieties, Comm. Algebra 26 (1998), 779-792.

9. J.-E. Pin and P. Weil, Profinite semigroups, Mal'cev products and identities, J. Algebra 182 (1996), 604-626.

10. J. Reiterman, The Birkhoff theorem for finite algebras, Algebra Universalis 14 (1982), $1-10$.

11. J. Rhodes, Infinite iteration of matrix semigroups, I. Structure theorem for torsion semigroups, J. Algebra 98 (1986), 422-451.

12. _ Infinite iteration of matrix semigroups, II. Structure theorem for arbitrary semigroups up to aperiodic morphism, J. Algebra 100 (1986), 25-137.

13. J. Rhodes and D. Allen Jr., Synthesis of the classical and modern theory of finite semigroups, Adv. in Math. 11 (1973), 238-266.

14. J. Rhodes and B. Steinberg, Join irreducible pseudovarieties, group mapping, and Kovács-Newman semigroups, LATIN 2004: Theoretical informatics, Lecture Notes in Comput. Sci., vol. 2976, Springer, Berlin, 2004, pp. 279-291.

15. Springer, 2009.

(Permanent address) CMUP, Dep. Matemática, Faculdade de CiÊncias, Universidade do Porto, Rua do Campo Alegre 687, 4169-007 Porto, Portugal. TEL.: +351 220402132; FAX: +351 220402108

E-mail address: jalmeida@fc.up.pt

Dept. of Mathematics and Statistics, Masaryk University, KotláŘská 2, 61137 Brno, Czech Republic

E-mail address: klima@math.muni.cz 